\title{
THE CASE FOR S STAR BINARIES
}

\author{
THOMAS B. AKE \\ Computer Sciences Corporation/Goddard Space Flight Center \\ Greenbelt, MD 20771, U.S.A.
}

\begin{abstract}
Several lines of evidence point to a scenario in which Tc-poor S stars are the cooler analogs to the Ba II stars, i.e. they are binary systems where the peculiar atmospheric composition of the primary star is due to mass accreted from a secondary star long ago. Cases have been found where such S stars have WD or main-sequence companions, but an increasing number are found to be in interactive, symbiotic-like systems. Evidence of wind shocks, gas-streaming, and/or accretion disks in these systems attest to the current proximity of the components and provide striking evidence that accretion can be an important mechanism in their chemical evolution. Interactive effects are not as prominent in Ba II stars, presumably because of the lack of a strong wind from the primary star.
\end{abstract}

\section{No text received}

\section{Discussion}

Frantsman: What is your opinion about the evolutionary phase of Tcpoor S stars? You mentioned that these stars and $\mathrm{Ba}$ II stars have similar properties (spectral characteristics, binarity). However it is known that their mean luminosities and effective temperatures are quite different.

Ake: The Tc-poor S stars are the cooler analogs of the Ba II stars. As the Ba II stars evolve up the giant branch, they become these $\mathrm{S}$ stars. The development of a wind, then, causes the $\mathrm{S}$ star binaries to show signs of interaction between the components, which is not normally seen in the Ba II star binaries. 
Frantsman: Why can't these stars be in the early-AGB phase, especially taking into account that for stars of a given mass this stage lasts considerably longer than the TP-AGB phase?

Ake: The S stars show enhancements of $s$-processing, except for Tc. Thus the surface abundance peculiarities indicate that the material was modified during the TP-AGB phase, but long ago.

Green: Just a technical question: What advantage is there to the GHRS over the FOS for the detection of white dwarf companions to the extrinsic S stars?

Ake: The GHRS doesn't have the scattered light problem of the FOS. 